\title{
Gamma-ray spectrometer (GRS) for lunar polar orbiter SELENE
}

\author{
Nobuyuki Hasebe $^{1}$, Eido Shibamura ${ }^{2}$, Takashi Miyachi ${ }^{1}$, Takeshi Takashima ${ }^{3}$, Masanori Kobayashi ${ }^{4}$, Osamu Okudaira ${ }^{1}$, \\ Naoyuki Yamashita ${ }^{1}$, Shingo Kobayashi ${ }^{1}$, Takeshi Ishizaki ${ }^{1}$, Kunitomo Sakurai ${ }^{1}$, Mitsuhiro Miyajima ${ }^{1}$, \\ Masayuki Fujii ${ }^{1}$, Keisuke Narasaki ${ }^{5}$, Shigeki Takai ${ }^{5}$, Katsuhiro Tsurumi $^{5}$, Hiroshi Kaneko ${ }^{6}$, \\ Michio Nakazawa $^{6}$, Kunishiro Mori ${ }^{7}$, Olivier Gasnault ${ }^{8}$, Sylvestre Maurice ${ }^{8}$, \\ Claude d'Uston ${ }^{8}$, Robert C. Reedy ${ }^{9}$, and Manuel Grande ${ }^{10}$ \\ ${ }^{1}$ Research Institute for Science and Engineering, Waseda University, 3-4-1, Okubo, Shinjuku, Tokyo 169-8555, Japan \\ ${ }^{2}$ College of Health Science, Saitama Prefectural University, 820, San' nomiya, Koshigaya, Saitama 343-8540, Japan \\ ${ }^{3}$ Institute of Space and Astronautical Science, Japan Aerospace Exploration Agency, \\ 3-1-1, Yoshinodai, Sagamihara, Kanagawa 229-8510, Japan \\ ${ }^{4}$ Nippon Medical School, 2-297-2, Kosugicho, Nakaharaku, Kawasaki 211-0063, Japan \\ ${ }^{5}$ Niihama Works, Sumitomo Heavy Industries Ltd., 5-2, soubirakicho, Niihama, Ehime 792-8588, Japan \\ ${ }^{6}$ Isesaki Works, Meisei Electric Co., Ltd., 2223 Naganumacho, Isesakishi, Gunma 372-8585, Japan \\ ${ }^{7}$ Clear Pulse Co., Ltd., 6-25-17, Chuo, Ohta-ku, Tokyo 143-0024, Japan \\ ${ }^{8}$ Centre d'Etude Spatiale des Rayonnements, Univ. Paul Sabatier, CNRS, 9 avenue Roche, 31400 Toulouse, France \\ ${ }^{9}$ Institute of Meteorites, Univ. of New Mexico, Albuquerque, NM, 87131-1126, USA \\ ${ }^{10}$ Institute of Mathematical and Physical Sciences, University of Wales, Aberystwyth, Ceredigion SY23 3BZ, Wales, UK
}

(Received April 1, 2007; Revised December 22, 2007; Accepted December 25, 2007; Online published April 9, 2008)

The high-precision gamma-ray spectrometer (GRS) on the lunar polar orbiter SELENE is designed to measure $200 \mathrm{keV}-12 \mathrm{MeV}$ gamma rays in order to determine elemental compositions of the lunar surface. The GRS consists of a large germanium $(\mathrm{Ge})$ crystal as a main detector and a massive bismuth germanate crystal and a plastic scintillator as anticoincidence detectors. The Ge detector is cooled by a Stirling cryocooler with its compressor attached to a passive radiator facing the cold space. The cooling system maintains the Ge detector below $90 \mathrm{~K}$ during the observation. The flight model of the GRS has achieved an energy resolution of $3.0 \mathrm{keV}$ (FWHM) at $1333 \mathrm{keV}$. Energy spectra obtained by the GRS will show sharp gamma-ray lines whose energies identify the elements and whose intensities determine the concentrations of the elements, permitting global mapping of the elemental abundances in the sub-surface of the Moon. The elemental maps obtained by the GRS with such high-energy resolution enable us to study lunar geoscience problems.

Key words: Moon, gamma-ray spectroscopy, chemical composition, lunar formation and evolution, SELENE (KAGUYA), GRS.

\section{Introduction}

To determine the spatial distribution of major and important trace elements in the lunar surface is an essential objective of lunar science. A lunar chemistry and the relative abundances of refractory and volatile elements provide clues to the conditions during the formation of the Moon. Combining planetological studies with elemental study can help us to improve our understanding of the evolution of the Moon and terrestrial planets. Gamma-ray spectroscopy is suited for measuring elemental composition in the lunar surface.

Planetary gamma-ray spectroscopy was first proposed by Arnold et al. (1962). $\mathrm{NaI}(\mathrm{Tl})$ scintillation spectrometers were boarded on the Apollo 15 and 16 missions, and lunar gamma rays were measured over about $20 \%$ of the lunar surface (Metzger et al., 1973). Elemental abundances in limited regions of $\mathrm{Fe}, \mathrm{Ti}, \mathrm{Mg}, \mathrm{K}$ and $\mathrm{Th}$ were measured by the Apollo gamma-ray observation (Bielefeld $e t$

Copyright (c) The Society of Geomagnetism and Earth, Planetary and Space Sciences (SGEPSS); The Seismological Society of Japan; The Volcanological Society of Japan; The Geodetic Society of Japan; The Japanese Society for Planetary Sciences; TERRAPUB. al., 1976). The data from this observation made it clear that lunar gamma-ray spectroscopy is a powerful and useful tool to determine elemental compositions and that numerical calculations used in interpreting the data were basically correct (Reedy, 1978). Recent gamma-ray observation conducted by Lunar Prospector has provided global maps of some major elements and natural radionuclides (Prettyman et al., 2006). Previous lunar missions, the Luna-10, the Apollo project and Lunar Prospector, have only conducted gamma-ray measurements using spectrometers with limited energy resolutions (Surkov, 1984; Bielefeld et al., 1976; Lawrence et al., 1998; Feldman et al., 1999). These scintillation detectors had large detection efficiencies because of their high atomic numbers, but their energy resolutions were not enough to distinguish many line gamma rays with various energies from the Moon. Thus, quantitative analyses of the energy spectra were difficult. In order to determine the elemental abundances precisely, a sophisticated gammaray spectrometer with both a high-detection efficiency and good energy resolution is required in a mixed gamma-ray field consisting of copious lines and continuum above the lunar surface. 
The idea of using a germanium (Ge) gamma-ray spectrometer with high-energy resolution for a planetary mission was proposed by Metzger and Arnold (1970) and Metzger et al. (1975). They discussed the advantage of combining high spectral resolution and excellent elemental identification. The first successful planetary mission carrying a Ge gamma-ray spectrometer was Mars Odyssey, which went into polar orbit around Mars in 2001. This gamma-ray spectrometer equipped with a Ge detector on Mars Odyssey was the first to show spectra with many narrow gamma-ray peaks (Boynton et al., 2004; Evans et al., 2007). The MESSENGER spacecraft now cruising to Mercury (Solomon $e t$ al., 2001) also carries a Ge gamma-ray spectrometer (Goldsten et al., 2007).

We review a high-resolution gamma-ray spectrometer (GRS) for the Japan's lunar explorer SELENE, the largest lunar orbiter since the Apollo missions. SELENE was launched by the H-IIA rocket on September 14, 2007. It will cover the whole lunar surface by its polar orbit at a nominal altitude of $100 \mathrm{~km}$ and observe the lunar surface with high precision by a three axis-stabilized attitude control. The instrument employs, for the first time in lunar exploration, a high-purity Ge crystal. The Ge detector is surrounded by BGO and plastic anticoincidence shields and cooled by a Stirling cryocooler. The SELENE-GRS measures gamma rays in the energy range of $200 \mathrm{keV}$ to $12 \mathrm{MeV}$ with high precision to determine the composition of the lunar surface. It will provide data on the abundance of many major elements over the entire lunar surface and possibly on water ice at the polar regions.

\section{Sources of Lunar Gamma Rays}

Galactic cosmic rays consist of various nuclei, mainly protons and alpha particles, with a very broad range in energy, but their average energy is of the order of $1 \mathrm{GeV}$, and their flux of about 3 particles $/ \mathrm{cm}^{2} \mathrm{~s}$ is nearly isotropic. They enter the lunar surface and generate nuclear cascades in the lunar sub-surface. The cascade particles producing gamma rays most effectively are neutrons. The secondary neutrons can escape into space or undergo further nuclear collisions. They may excite stable nuclei in the lunar soil to higher energy levels by nonelastic reactions, or they may scatter off nuclei and lose energy until they are thermalized and captured, producing capture gamma rays. Most of the abundant elements provide line gamma rays from these processes. Cosmic ray-produced radioactive nuclei, such as ${ }^{26} \mathrm{Al}$ or ${ }^{7} \mathrm{Be}$, also produce gamma rays.

In addition to the gamma rays being emitted by the above processes, natural radioisotopes in the ${ }^{232} \mathrm{Th}$ and ${ }^{238} \mathrm{U}$ decay chain and ${ }^{40} \mathrm{~K}$ also generate line gamma rays. The intensities of $2615 \mathrm{keV}$ by the deexcitation of ${ }^{208} \mathrm{Tl}$ in the thorium chain and of $1461 \mathrm{keV}$ by the decay of ${ }^{40} \mathrm{~K}$ are sufficiently high to enable them to be detected by a gamma-ray spectrometer. These gamma rays are good tracers of Th and $\mathrm{K}$, respectively (Lawrence et al., 1998).

A gamma-ray spectrometer in an orbit around the Moon can measure the characteristic energies of such lines since there is no atmosphere on the Moon. The energy and intensity of the lines identify the species of elements and their abundance in the lunar surface, respectively (Reedy et al.,
1973; Reedy, 1978; Evans et al., 1993; Yamashita et al., 2008).

\section{Role of the GRS in the SELENE Mission and Scientific Objectives}

The Moon is our cornerstone in the study of primitive planetary evolution. As a matter of course, the Moon is in itself the object of interest. To date, dozens of spacecraft have been sent to the Moon. With the returned Apollo and Luna samples collected in situ, the Moon became the most well-known planetary body after the Earth. Lunar Prospector (Binder, 1998), SMART-1 (Foing et al., 2007), and Clementine (Nozette et al., 1994) globally surveyed the lunar surface and provided geochemical and petrogenetic pictures of the Moon.

One of the scientific objectives of SELENE is to provide global maps of the Moon's chemical and mineralogical distribution, its surface and sub-surface structure, and its gravity and magnetic field, and to provide data elucidating the origin and evolution of the Moon. The GRS on SELENE is the first gamma-ray spectrometer that fulfills the criterion required from lunar science: the unique identification of elements and quantitative analysis of them with high precision and sensitivity. The GRS has an excellent energy resolution, as will be shown later in this article, and it has the capability to uniquely identify many surface elements, such as $\mathrm{O}, \mathrm{Mg}, \mathrm{Al}, \mathrm{Si}, \mathrm{K}, \mathrm{Ca}, \mathrm{Ti}, \mathrm{Fe}, \mathrm{U}$, and $\mathrm{Th}$, to provide global maps of the Moon. In addition, the GRS will search for the possibility of the presence of water ice in the permanently shaded regions (Kobayashi et al., 2002; Berezhnoy et al., 2003a).

Gamma-ray observation by the GRS will be conducted together with other 14 instruments on board SELENE, including a X-ray spectrometer (XRS), multi-band imager (MI), spectral profiler (SP), terrain camera (TC), lunar radar sounder (LRS), laser altimeter (LALT), lunar magnetometer (LMAG), relay satellite, and VLBI radio source (RSAT+VRAD), with the aim of measuring the lunar gravity field (Sasaki et al., 2003). The GRS's results will be inter-calibrated and be verified with those of the XRS, MI, and SP. The elemental distributions precisely measured for major elements of rocks, radioactive elements, and volatile and refractory elements, will provide basic knowledge of the Moon and will contribute directly or indirectly to the progress of lunar science. Relating the data obtained with the other instruments will also improve significantly our current understanding of the Moon as described in the next section.

\subsection{Science of the Moon}

3.1.1 Significance of understanding the Moon The Moon could have originated following the impact of a Mars-sized projectile onto the Earth in its earliest history. It is suggestive that the Moon accreted very rapidly, perhaps from debris resulting from the cataclysmic collision. This was likely followed by planetary scale melting and the formation of a magma ocean, which are the mechanisms behind the first stage of lunar differentiation. It is known that the Moon preserves records of the accretional impacthistory of the inner solar system and of the remnants of the primordial crust and early differentiated mantle; this is in 
contrast with the Earth, which has been significantly modified by tectonics. A detailed understanding of the Moon will be critical in solving problems on other planetary bodies, including the Earth. Thus, the Moon will always remain a cornerstone in our understanding of the Solar System. Precise measurements of the surface abundance of elements will facilitate our understanding of the planetary evolution.

3.1.2 Formation of the lunar crust and its dichotomy The Moon could have undergone extensive melting in its early history, leading to the formation of (an) anorthositic plagioclase crust(s) by floatation to the surface of this magmatic ocean, as has been inferred from Apollo samples. The extension and the nature of the processes occurring in the lunar magma ocean are still open to discussion (Shearer and Papike, 1999; Jolliff et al., 2000; Warren, 2005). The recent analysis of the Dhofar 489 lunar meteorite suggests the existence of magnesian anorthosite in the lunar farside that fills the gap between the ferroan anorthosite and $\mathrm{Mg}$ suite (Takeda et al., 2006) and that Apollo and Luna samples are not representative of the whole moon. The integration of lunar meteorite analysis and remote sensing data contributes to determining the source of lunar meteorites and is indispensable to improving the accuracy of elemental composition data, especially $\mathrm{U}, \mathrm{Th}, \mathrm{K}, \mathrm{Ca}, \mathrm{Ti}$, and $\mathrm{Mg} \#(=\mathrm{Mg} /(\mathrm{Fe}+\mathrm{Mg}))$.

3.1.3 Crust and mantle composition The abundance of refractory elements, e.g., U and Th, provides particularly important information about the degree of fractionation of the lunar crust. $\mathrm{U}, \mathrm{Th}$, and $\mathrm{K}$ abundances in the lunar crust are also important as constraints on the present magnitude of radionuclide heating sources. $\mathrm{K} / \mathrm{U}$ and $\mathrm{K} / \mathrm{Th}$ ratios are also useful for the estimation of the abundances of $\mathrm{K}$ and other moderately volatile elements.

To determine the composition of the lunar mantle is a major goal of geochemical study of the Moon. The SELENE-GRS/XRS/SP/MI can perform chemical analysis of deep materials derived from partial melting of the mantle and from excavating large basins, such as the South PoleAitken. In addition, lunar magmatism changes with time and reflects mantle heterogeneities; as such, evolution can be tracked through various basalt compositions at the surface. Some elements, such as Ti, for example, which are considered to be highly suggestive of inner source regions of the mare materials, can be detected with high sensitivity by the GRS. We expect that interesting data on the composition of the mantle will be inferred from the analysis.

3.1.4 The interior of the Moon Our knowledge of the lunar structure is still deficient. We do not know what the bulk composition of the Moon is, and what the structural, compositional, and thickness variations of the lunar crust are. Apollo seismic data are clustered close together on the near-side and represent only a limited lunar terrain. The data on variations in crustal structure and thickness are limited. We have imprecise and ambiguous data for the deeper lunar mantle and core. The global observation by the GRS provides data on the chemical abundances of major elements in the lunar surface with a higher precision than ever done before, and the integration of data obtained by the GRS and the other instruments, such as LRS, RSAT+VRAD, and the Apollo seismic data (Nakamura et al., 1982) furthers our understandings for the interior of the Moon.

3.1.5 Volatile reservoirs and volatile transport The GRS observation will address problems having to do with volatiles, mainly $\mathrm{H}_{2} \mathrm{O}$ and possibly $\mathrm{SO}_{2}$, at the lunar surface (Berezhnoy et al., 2003b). The lunar polar deposits are thought to be dominated by layered deposits consisting of a mixture of dust and $\mathrm{H}_{2} \mathrm{O}$ (Feldman et al., 2000, 2001; Nozette et al., 2001). Current orbital data by Feldman et al. (2001) and Lawrence et al. (2006) which revealed a threefold enhancement of $\mathrm{H}$ content in the polar regions as compared to equatorial regions are now open to interpretation regarding the nature and the extent of these H-rich deposits. The more recent Earth-based observations show no evidence for thick deposits, but they do not rule out disseminated ice in the regolith (Campbell et al., 2006). More detailed orbital mapping of chemical composition and mineralogy in pyroclastic deposits is needed. Conclusive orbital data representing $\mathrm{H}_{2} \mathrm{O}$ ice deposits are required. We have to clearly determine whether $\mathrm{H}_{2} \mathrm{O}$ ice deposits exist at the lunar poles or not. We also have to clearly answer questions on what the lateral and vertical extents of these deposits are, how long these deposits have been formed, and what transport processes are active for volatiles on the lunar surface. The GRS observation will be able to measure the mixture of dust and volatiles to a depth of several tens of centimeters. Direct detection of hydrogen can be made by the prompt gamma-ray line $(2223 \mathrm{keV})$. In addition, during a possible extended mission, in which SELENE is planned to orbit at an altitude of $50 \mathrm{~km}$, information about water ice deposits will be obtained by the GRS.

\subsection{Utilization of the Moon}

The utilization of natural resources available from the Moon is essential for the manned exploration of the solar system in the future. Metals such as $\mathrm{Fe}$ and $\mathrm{Al}$ are inevitably important to construct a lunar base. The production of $\mathrm{H}_{2} \mathrm{O}$ and $\mathrm{O}_{2}$ is critical to human activity on the Moon. Other materials, such as casting ones, are necessary to develop permanent human settlements. Massive shielding materials must protect inhabitants from cosmic radiation and meteorite impacts.

Previous unmanned lunar missions (Nozette et al., 1994; Binder, 1998) provided valuable information on materials available on the Moon: global data sets gave the distribution of many potential lunar resources, such as plagioclase, pyroxene, ilmenite, and olivine (Lucey, 2004). The data can also be used to determine the $\mathrm{FeO}$ and $\mathrm{TiO}_{2}$ contents with high spatial resolution (Lucey et al., 2000; Gillis et al., 2003, 2004; Lawrence et al., 2002).

It is known that lunar surface materials are a source of the raw materials that produce structural metals, oxygen, silicon, glass, and ceramic products. Ilmenite, including $\mathrm{Ti}$, can serve as a "supplier" of oxygen and is found in volcanic lava flow areas. The maps of Ti distribution in lava flow areas were produced using data collected by the Clementine and Lunar Prospector (Lucey et al., 2000; Gillis et al., 2003; Prettyman et al., 2006). In addition, ${ }^{3} \mathrm{He}$ is probably very important since it will be used as a fuel in nuclear fusion reactors in the future. The abundance of ${ }^{3} \mathrm{He}$ was mapped in association with $\mathrm{TiO}_{2}$ and surface maturity (Johnson et 


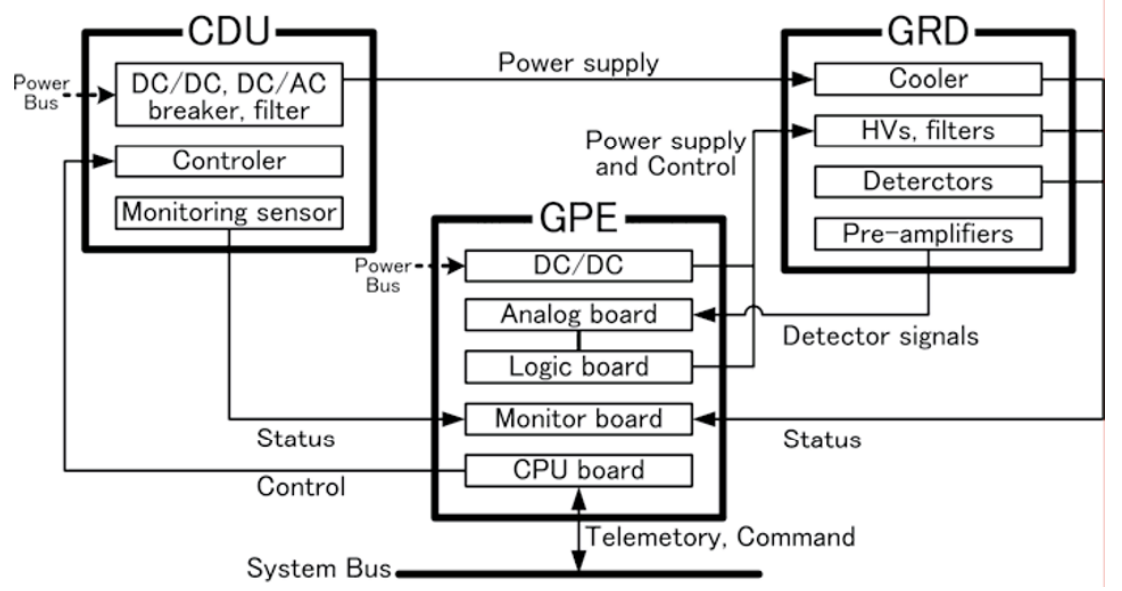

Fig. 1. Block diagram of the GRS system. The system is composed of three subsystems: GRD, CDU, and GPE.

\section{al., 1999).}

The observation by SELENE focuses on creating precise maps of water ice, permanent polar shadow/sunshine areas, and mineral distribution, and on making a digital elevation model of the lunar poles. As discussed in Section 3.1, the possible existence of hydrogen can also be confirmed by observing gamma rays induced from hydrogen. The amount of hydrogen in or beneath a regolith layer will be quantitatively estimated and mapped using the SELENEGRS observation. SELENE data will provide data on the best site for a lunar base in the future.

\section{Instruments}

The gamma-ray spectrometer (GRS) serves to detect line gamma rays in order to identify elements, their concentration, and their distribution on the Moon. The GRS system consists of three subsystems: a gamma-ray detector (GRD), cooler driving unit (CDU), and gamma-ray and particle detectors electronics (GPE). The block diagram is shown in Fig. 1. The GRD subsystem placed outside the spacecraft contains a germanium detector with a cryocooler and radiator, anti-counters, preamplifiers, high-voltage power suppliers and their filters. The CDU installed inside the spacecraft is a $55-\mathrm{W}$ power supply for driving the cryocooler. This is controlled by the GPE subsystem. The GPE contains ana$\log$ and digital circuit boards for processing and reducing data as well as computer-processing-unit (CPU) boards for data handling, transmitting, and command decoding. The GPE subsystem is shared with the CPS (charged particle spectrometer) system.

Section 4.1 mainly deals with the detector units of the GRD, which form a major subsystem of GRS. The GPE and CDU are described in Section 4.2, and the performance of the GRS is summarized in Section 4.3, in which the spatial resolution is also discussed.

\subsection{Gamma-ray detector}

The configuration of GRD is shown in Fig. 2 and the overall view of GRD is represented in Fig. 3. The principal detector of GRD is a highly-purified Ge detector that is cooled with a Stirling cryocooler to keep the GRD below $90 \mathrm{~K}$. The Ge detector is a cylindrical, with a diameter of $65 \mathrm{~mm}$ and a height of $77 \mathrm{~mm}, 252-\mathrm{cm}^{3}$, single-ended coaxial type. The Ge crystal is encapsulated with a highvacuum tight aluminum canister. The canister of the Ge crystal is supported by a diaphragm-type support, a ringshaped support, and a cantilever-typed support at the time of launching, as shown in Fig. 4. At cryogenic temperature, the four claws of the ring-shaped support are released by contraction to reduce heat conduction.

The Ge detector is surrounded with scintillation counters: a BGO counter and a plastic counter. The former is horseshoe shaped, as shown in Fig. 5. It is composed of two symmetric-shaped crystals $\left(\mathrm{BGO}_{1}\right.$ and $\left.\mathrm{BGO}_{2}\right)$ which are bonded optically. This surrounds the Ge crystal, as shown in Fig. 2. The thick part of the BGO crystals (maximum $4 \mathrm{~cm}$ ) faces the body of the SELENE satellite in order to veto events of gamma rays emitted from materials of the satellite body. The opposite side is open to view the nadir of the lunar surface. The BGO crystals also serve as an active anticounter that tags events occurring in both the Ge and BGO detectors. By this configuration, the photoelectric events that directly measure the energies of gamma rays from the lunar surface are effectively observed, while background events are inhibited. The BGO crystals are viewed with two photomultipliers.

The plastic counter, $5 \mathrm{~mm}$ thick, is used to veto events generated by charged particles (see Fig. 6). A single photomultiplier serves to count scintillation light from the plastic scintillation counter.

The Ge crystal must be cooled; the detector is operated below $\sim 90 \mathrm{~K}$. This was achieved by a Stirling-cycle cryocooler. The cooler comprises two parts: a compressor and a cold head (Fig. 7). It normally operates for more than $60,000 \mathrm{~h}$. The cooling system is coupled with the Ge crystal via a thermal link that is described below. The details of the cooling system have been reported elsewhere (Kobayashi, 2005).

Since the Stirling cooler can be a source of microphonic noise due to a mechanical vibration, it is important to mechanically isolate it but still to thermally couple the cold tip with the Ge crystal. This problem is successfully solved using a relatively quiet cooler and a flexible link that is a bundle of 15-folded copper sheets as shown in Fig. 8. The link is effective in suppressing the noise level to an extent that 


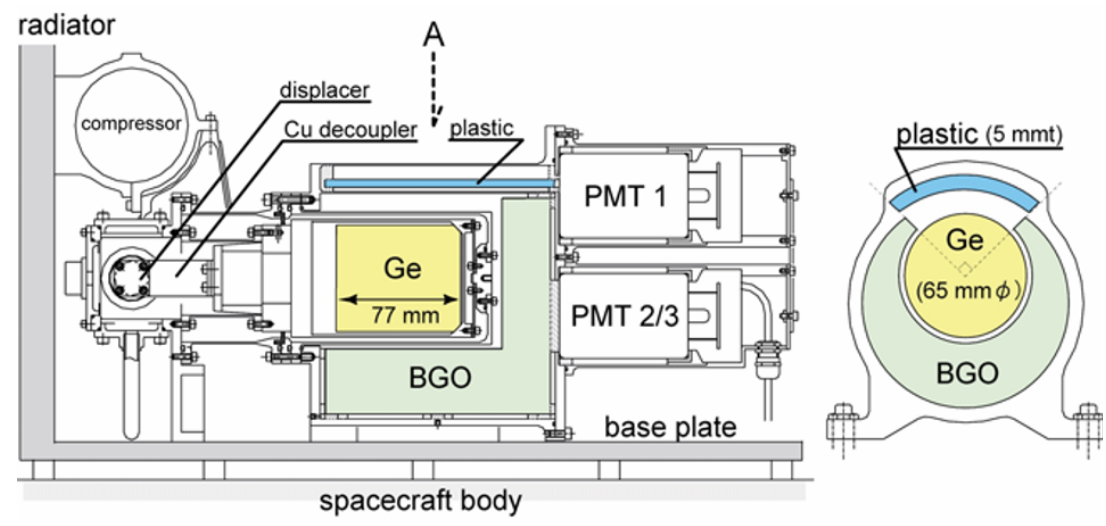

Fig. 2. Schematic drawing of GRD. Incident gamma rays from the Moon come through the plastic scintillator and viewed by a photomultiplier (PMT1). The space around the Ge crystal, the cold-tip of the displacer and thermal link $\left(\mathrm{Cu}\right.$ decoupler), is evacuated. The $\mathrm{BGO}$ crystals $\left(\mathrm{BGO}_{1}\right.$ and $\left.\mathrm{BGO}_{2}\right)$ are coupled with two PMTs.

the energy resolution of GRD is good. In order to suppress possible thermal noise at the first stage of the front-end amplifier, it is mounted on a small circuit board that is placed close to the Ge crystal for cooling.

Since the mechanical cooler is driven by an input power of $52 \mathrm{~W}$ at maximum, the heat generated in the cooler has to be removed towards the cold space through a passive radiator. The radiator with optical solar reflector (OSR) has a large area of $39 \times 60 \mathrm{~cm}^{2}$ facing towards the cold space. The temperatures of the Ge and BGO crystals are almost constant, although thermal input from the lunar surface heavily depends on the local time of the Moon. According to a study of heat flow, the amount of thermal inflow to the $\mathrm{Ge}$ detector is estimated to be $\sim 2 \mathrm{~W}$, as represented with a chart in Fig. 9. In order to increase the cooling efficiency of the Stirling cooler, the inflow of thermal radiation is attenuated by reflection methods: a passive radiator covered with OSR of absorption coefficient $\alpha=0.2$ and emissivity $\varepsilon=0.8$. In addition, the entire surface of the GRD is covered with multi-layer insulators $(\alpha=0.93$ and $\varepsilon=0.85)$. The radiation exhaust enables the Ge crystal to be kept below $\sim 90 \mathrm{~K}$.

The mechanical characteristics of the GRS were tested with vibrations in three dimensions. According to the results of the analysis, the GRS has fulfilled criteria of mechanical disturbance during a launch of H-IIA rocket (Kobayashi et al., 2005). Moreover, the effects due to electromagnetic compatibility were tested and any adverse influence upon other instruments was found as negligible.

According to the thermal analysis, the Ge detector is well kept at around $80 \mathrm{~K}$. This is confirmed in the measurements shown in Fig. 10. The temperature at the Ge detector is measured as a function of time elapsed after the cooling system sets on with a driving voltage of $10 \mathrm{~V}$. Its driving voltage was turned up to $17 \mathrm{~V}$ at $12 \mathrm{~h}$ after cooling starts, at a discontinuity point of cold-tip temperature in Fig. 10. It takes about $24 \mathrm{~h}$ to arrive at $80 \mathrm{~K}$ from room temperature. This is achieved in the case that the heat capacitance is $2 \mathrm{~W}$, which corresponds to $52 \mathrm{~W}$ in terms of the input power to the cryocooler.

A heater to anneal the Ge crystal is equipped in the GRD system to recover the good energy resolution when it is deteriorated by radiation damage. It is expected that the

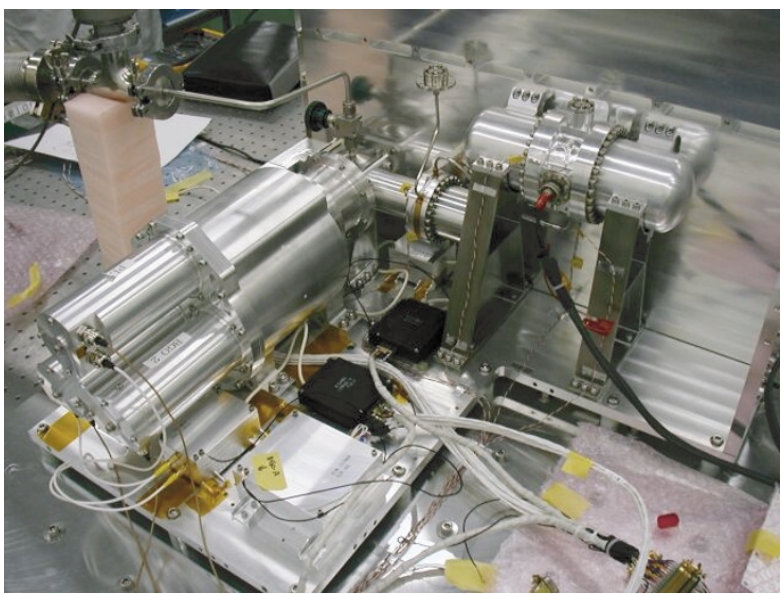

Fig. 3. Photograph of the flight model of the GRD assembly.

annealing process can be performed when the resolution becomes poor in orbit.

The energy resolution of the Ge detector is measured using a ${ }^{60} \mathrm{Co}$ standard gamma-ray source. In Fig. 11, a single spectrum of ${ }^{60} \mathrm{Co}$ is presented. The two peaks corresponding to line gamma rays at 1173 and $1333 \mathrm{keV}$ are clearly shown. In the inset, the FWHM at the 1333-keV gamma peak is confirmed to be $3.0 \mathrm{keV}$. This magnitude is affected by mechanical disturbance as well as by the functions of the electronic circuits. The former problem is cleared up by the procedures described above. The latter is solved by adjusting the time constants of the amplifiers used in the electronic circuits. This is described in the next section.

\subsection{Electronics}

4.2.1 Analogue circuit The GRS has two highvoltage power supplies for the Ge crystal and the photomultiplier tubes (PMTs). These are DC-DC converters that provide Ge with regulated $0-3.5 \mathrm{kV}$ DC and PMTs with $0-1.5 \mathrm{kV}$ DC.

In ground tests, the nominal bias voltage for the Ge detector is $3.1 \mathrm{kV}$, at which voltage depletion volume is saturated enough and the saturation of carriers produced is also attained. We will adopt $3.1 \mathrm{kV}$ for nominal $\mathrm{HV}$ at the beginning of the operation in lunar orbit, but it may be elevated up 


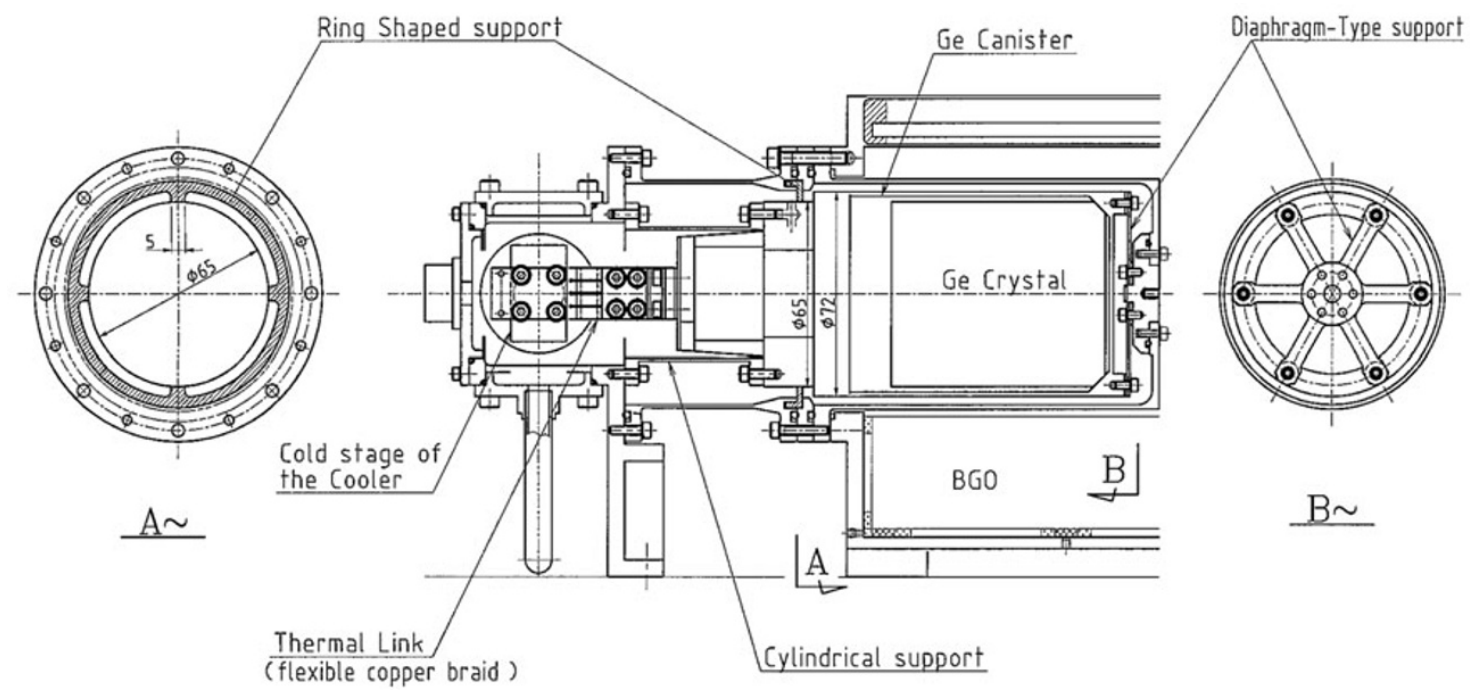

Fig. 4. Supporting methods and thermal coupling to the Ge crystal. The canister containing the Ge crystal is supported by a diaphragm-type support, a ring-shaped support, and a cantilever-typed support. At cryogenic temperature, the four claws of the ring shaped support are released by contraction to reduce heat conduction.

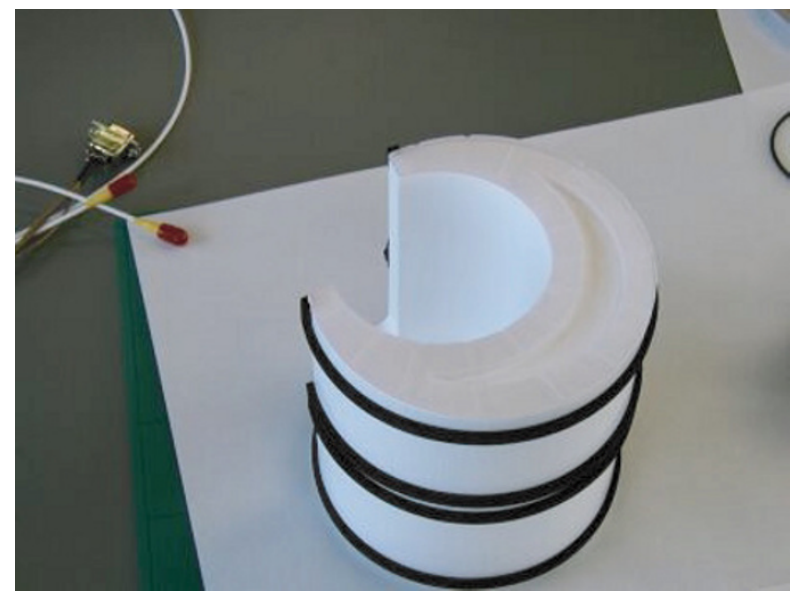

Fig. 5. BGO crystal covered with white paint. The BGO detector surrounds the Ge crystal to veto events that are induced by gamma rays and charged particles through the crystal. This serves as an anti-counter to the Compton scattering inside the Ge crystal and lowers the background gamma rays from the satellite.

to $3.5 \mathrm{kV}$ when the charge is not completely collected due to radiation damage in space. The bias voltage for PMTs coupled to scintillators is nominally $1.1 \mathrm{kV}$ in ground tests, and it may be elevated up to $1.5 \mathrm{kV}$ due to the deterioration of the PMT or the scintillators. These high voltages are filtered with a C-R filter network that suppresses the ripple of the primary voltage. The high-voltage power supplies and the filters are placed near these sensors on the outside of the satellite.

The amplifier for Ge signals includes a charge-sensitive preamplifier (PA) and a linear amplifier (LA). A front-end circuit board consisting of a field effect transistor (FET), the feed-back components of the PA, is located on the canister of the Ge sensor and is operated at a temperature of around $130 \mathrm{~K}$. The remainder of the PA is located outside the cryostat of Ge. The signal from the PA is transmitted through a 5-m cable into the satellite body and is fed to the shaping

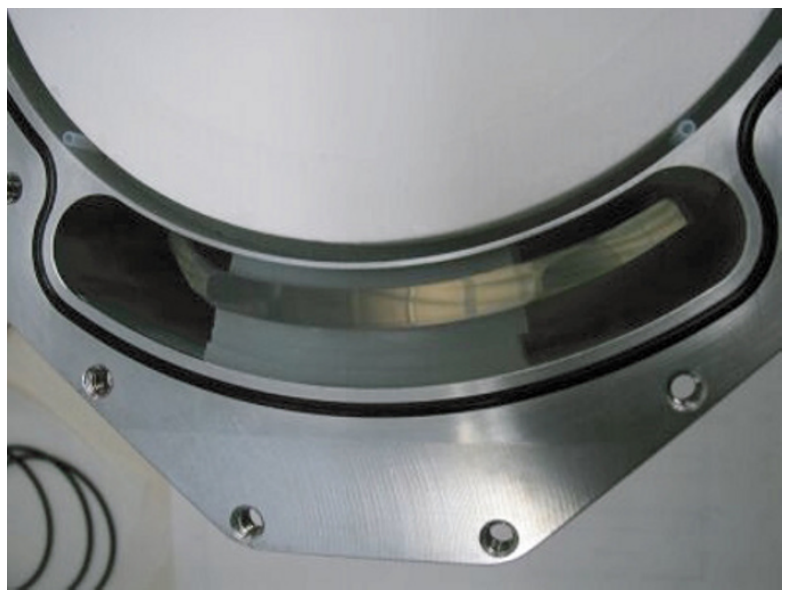

Fig. 6. Plastic scintillator accommodated in the outer aluminum package.

amplifier after a differential input buffer and a gain control circuit in GPE. In Fig. 12, a block diagram for the analog signal processing of the Ge detector after the PA output is shown. The gain can be digitally controlled by a command from the Earth ground station. The pulse shaping is of the uni-polar and semi-Gaussian type with a time constant of $2.0 \mu \mathrm{s}$. This time constant has been determined to minimize the electric noise under the presence of mechanical vibration due to the Stirling refrigerator. The shaped signal is fed to a base-line restorer and is fed to two amplifiers: a low-gain line that covers the energy range from $200 \mathrm{keV}$ to $12 \mathrm{MeV}$ and one that covers the energy range between $200 \mathrm{keV}$ and $3 \mathrm{MeV}$. The signal of each amplifier is fed to an analogue-to-digital converter (ADC) through a peak-hold circuit. If the signal saturates over the maximum level, the discrimination signal is fed to a logic circuit and inhibits the subsequent pulses for $300 \mu \mathrm{s}$. This duration is needed for the base line to recover. The energy resolution was $3.0 \mathrm{keV}$ (FWHM) for $1333 \mathrm{keV}$ gamma rays from ${ }^{60} \mathrm{Co}$ (see Fig. 11). 


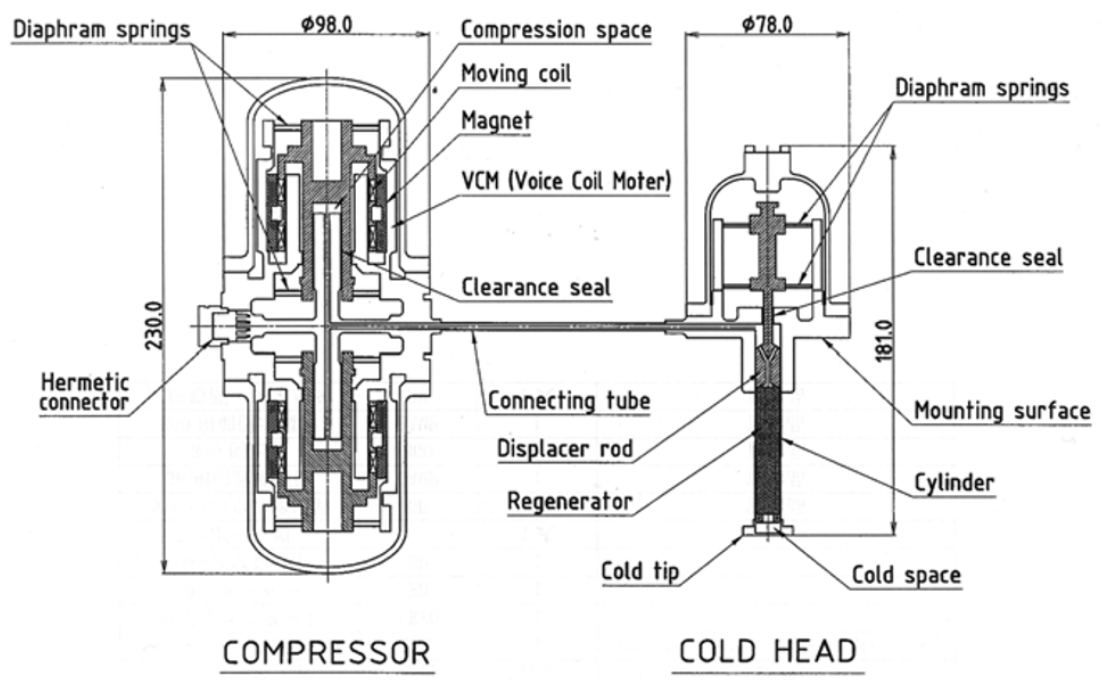

Fig. 7. Cryocooler system. The Ge detector is connected to a cold tip of the cold head through thermal link.

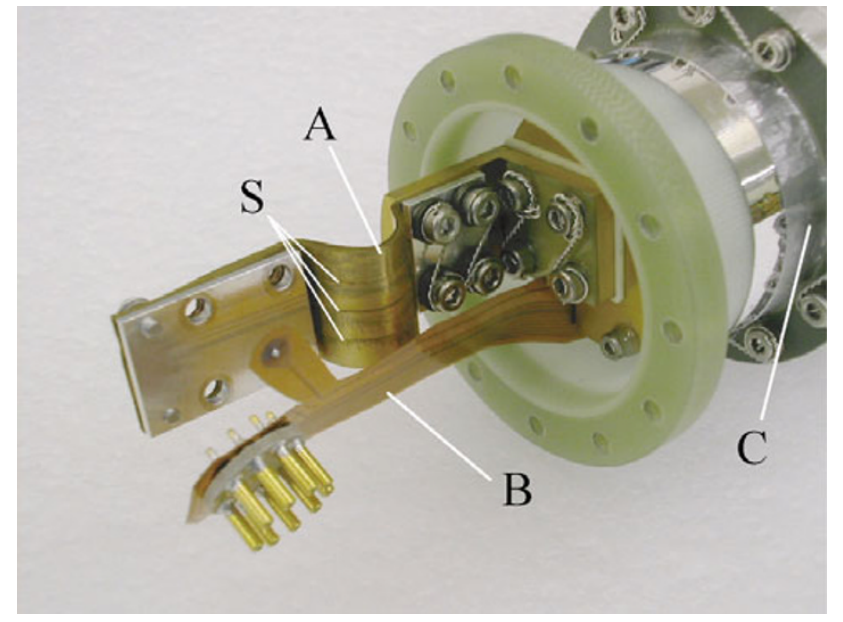

Fig. 8. Connection from the Ge crystal. A represents the thermal link; $\mathrm{S}$, slits on the link; $\mathrm{B}$, the signal lines from the amplifier; $\mathrm{C}$, a packing material.

The scintillation signals from $\mathrm{BGO}_{1}, \mathrm{BGO}_{2}$, and the plastic scintillator are processed by PMTs, preamplifiers, and linear amplifiers, respectively, with a gain-controller and pulse discriminators. The $\mathrm{BGO}_{1}$ and $\mathrm{BGO}_{2}$ signals are summed up after the gain is adjusted so that the difference between the two PMTs' outputs is minimized. Figure 12 also shows a block diagram for analogue signal processing of BGO scintillators after the PA output. The summed BGO signal is shaped in a bipolar pulse with a time constant of $3.7 \mu \mathrm{s}$. Since the BGO detector is so large and massive, events will frequently occur with excessively large energy loss. We chose bipolar shaping to shorten the recovery time to the level of the baseline in the case of excessively large signals. With this shaping, we confirmed that the BGO signals can be measured in the presence of $500-\mathrm{Hz}$ light pulses equivalent to $200 \mathrm{MeV}$ deposited energy. The energy resolution of the BGO detector is about $10-14 \%$, depending on the irradiation condition, at $662 \mathrm{keV}$ gamma rays from ${ }^{137}$ Cs. These shaped signals are fed to a peak-hold cir-

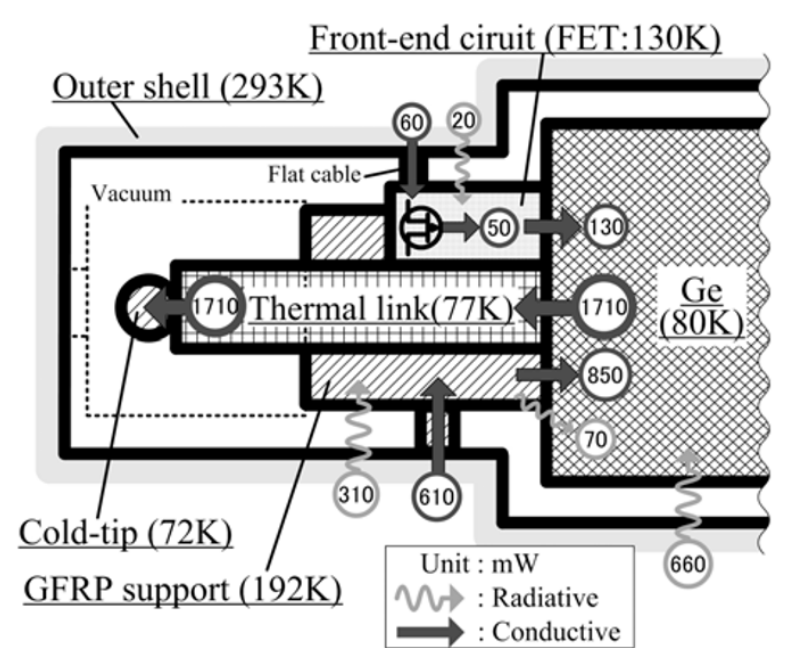

Fig. 9. Heat flow. The operational temperature of the Ge crystal is kept at 80-90 K.

cuit followed by ADC used in BGO-MODE, and they are also fed to five comparators connected in parallel. These comparators have five different discrimination levels, corresponding to approximately 100-, 300-, 700-, 3000-, 9000$\mathrm{keV}$ gamma rays. When the anti-coincidence function is engaged in GE-MODE, one of these signals is used to inhibit the Ge signal. These comparators are regarded as a five-channel analyzer in GE-MODE. The result is recorded as a counting rate for $17-\mathrm{s}$. The signal from the plastic scintillator is processed by a circuit similar to those for BGOs. This plastic signal is used to veto the Ge counter in GEMODE when it fires.

When a gamma-ray burst is detected, the spectrometer operates in a special mode called BURST-MODE, in which the sum signals of the $\mathrm{BGO}_{1}$ and $\mathrm{BGO}_{2}$ are sent to five comparators, and the outputs are sent to five counters; in addition, Ge signals are recorded in PHA for $1 \mathrm{~s}$. The counts are recorded every $10 \mathrm{~ms}$ for approximately the first $15 \mathrm{~s}$ from the occurrence of the burst. 


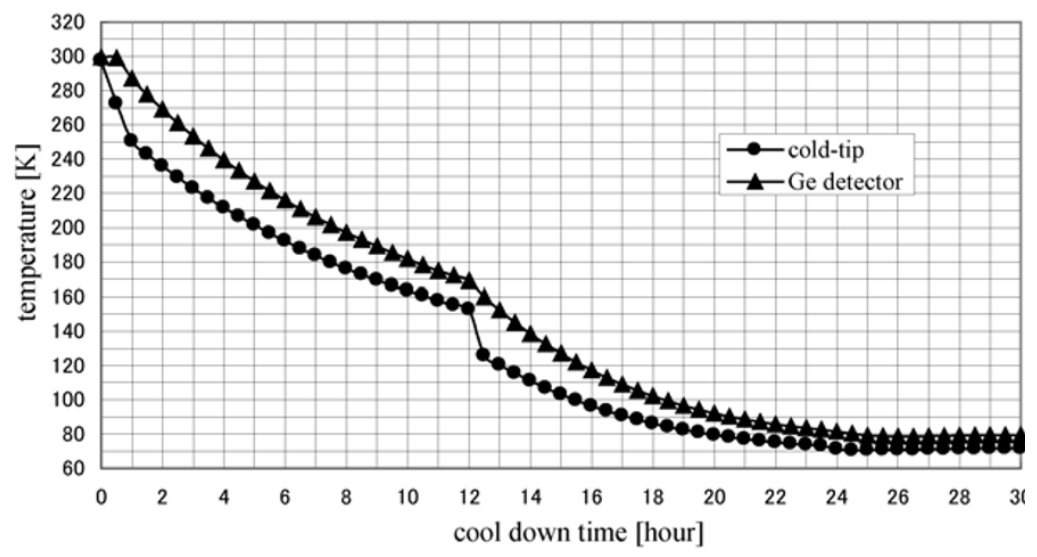

Fig. 10. Time profile of temperature variation of the Ge crystal and the cold tip after CDU sets on. It takes $24 \mathrm{~h}$ to arrive at the saturated heat balance. The driving voltage of CDU was changed from 10 to $17 \mathrm{~V}$ at $12 \mathrm{~h}$ after the cooling started.

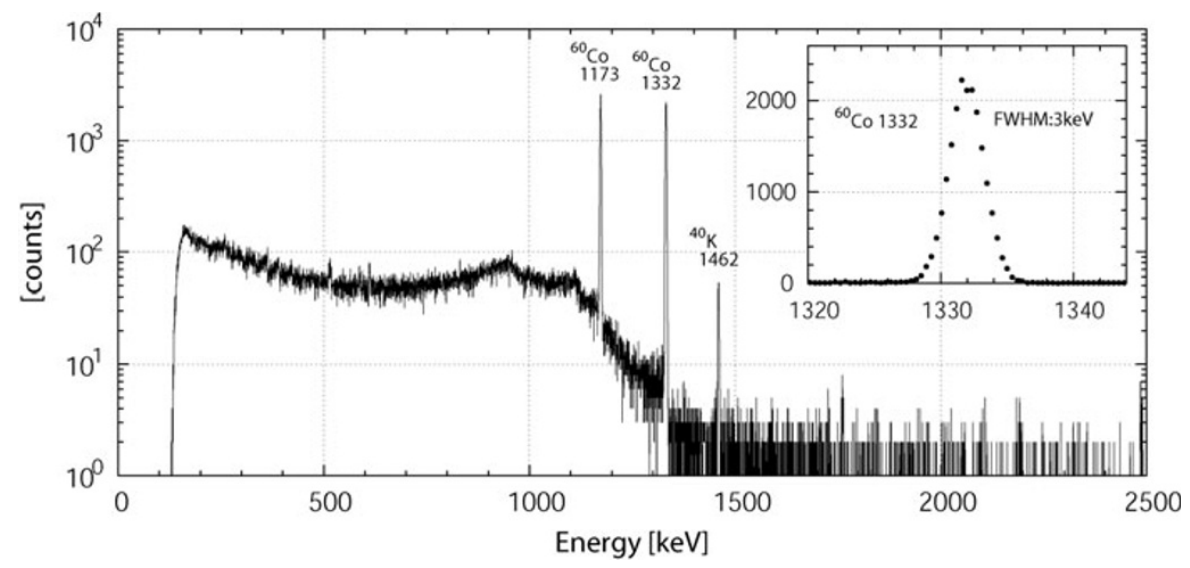

Fig. 11. A simple energy spectrum of gamma rays measured by the Ge detector for ${ }^{60} \mathrm{Co}$. Two lines from ${ }^{60} \mathrm{Co}$ and a single line of natural ${ }^{40} \mathrm{~K}$ are seen. The peak width at $1333 \mathrm{keV}$ is confirmed to be $3.0 \mathrm{keV}$ in FWHM.

4.2.2 Digital circuit Signals from the linear amplifiers and the comparators are fed to a logic circuit board, which has three floating programming gate arrays (FPGAs) and a CPU set (three-redundancy SH-3 RISC CPUs, adopting the majority rule preventing it from an error by singleevent-upset). It also has a pair of dual-port RAMs for making histograms and passing data to the CPU set. These play a role in the digital-data processing of the detectors' output signals and the housekeeping (HK) data of the GRS and also in decoding and executing commands from the ground station.

The FPGA overwatches outputs from the comparators and also varies flags of anti-coincidence enable/disable to determine if the Ge detectors' outputs are to be acquired. When the FPGA finds an event to be processed, it executes a sequence of data acquisition. In the sequence, the FPGA controls peak-hold circuits, a multiplexer, and an ADC for $\mathrm{AD}$ conversion of the Ge detectors' outputs. The FPGA subsequently implements an add-one data process (adding a number content corresponding to its memory address) to make a histogram in one side of the dual-port RAM bank. The memory bank is alternately switched every $17 \mathrm{~s}$ by a control signal from the FPGA so that the Ge detectors' outputs are integrated into two 8192-channel histograms (8-bit counter each channel) and 14 counters (16-bit counter each) every $17 \mathrm{~s}$. While the FPGA is making a histogram in one RAM bank, the CPU set reads the histogram data from the other side of another RAM bank. It subsequently divides the data into 17 packets and sends them to the spacecraft system.

In addition to the histogram, comparators' outputs are also counted by the FPGA. These data indicate event numbers stored in 14 counters, such as events from the detectors, over-range events from each detector, and dead time of the electronics. The dead time is measured by the digital circuit. The ADC for the Ge detector is forced to be inhibited in any case for $200 \mu \mathrm{s}$ after normal events and for $300 \mu \mathrm{s}$ after over-range events. Thus, the dead time is measured by counting events of the Ge detector and the anti-coincidence detectors. Another FPGA controls the gains of the amplifiers, high voltages of the detectors, and output power CDU according to commands through the CPU.

The other FPGA on the monitor board collects data on temperatures and other information on HK. It digitizes the data of the 256-channel ADCs and stores them in its resisters. They are transmitted to the ground via the CPU set as HK data.

4.2.3 CDU The CDU (Cooler Driving Unit) subsystem, a power supply for the Stirling cooler, has a DC to $\mathrm{AC}$ converter that was designed to be compatible with 


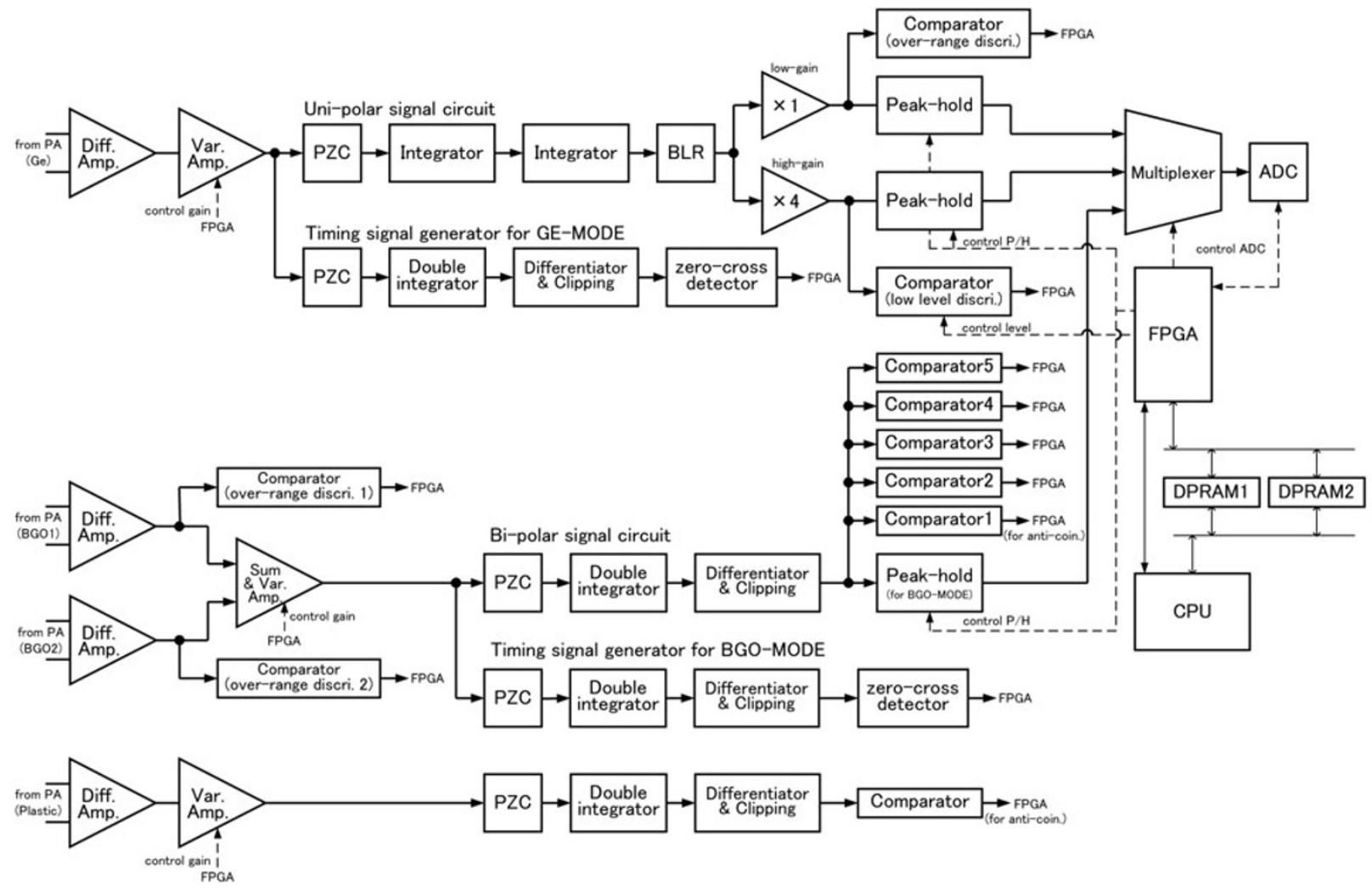

Fig. 12. Block diagram of analog signal processing of the Ge detector, the BGO, and the plastic scintillators of SELENE GRS.

electromagnetic specifications. The size and weight are $400 \times 360 \times 59 \mathrm{~mm}^{3}$ and $4.3 \mathrm{~kg}$, respectively. The averaged input power is approximately $76 \mathrm{~W}$ when the output power of $52 \mathrm{~W}$ is coupled by the cooler and the output voltage is $17 \mathrm{~V}$. Since the CDU is directly supplied by its own primary line, independent from GRD and other instruments, it is able to drive the cooler to cool the Ge crystal while the GRD subsystem is turned off. Its output level is controlled from 0 to $17 \mathrm{~V}$ AC by the GPE subsystem.

\subsection{Performance of GRS}

The characteristics of GRS are summarized in Table 1. The GRS looks at the nadir within a precision of better than 1 degree. Mission data cover an energy range from $200 \mathrm{keV}$ to $12 \mathrm{MeV}$. The energy resolution of the flight model is $3.0 \mathrm{keV}$ at $1333 \mathrm{keV}$. The accumulation time of energy spectra is $17 \mathrm{~s}$, which corresponds to a ground track of $26 \mathrm{~km}$ during the period at a nominal altitude of $100 \mathrm{~km}$.

Since the SELENE is stabilized on three axes and the GRS continues to face the lunar surface, the spatial resolution of the GRS and the shape of the footprint depends on a spatial response function (Lawrence et al., 2000) and the detection efficiency of the GRD. The spatial response function is expressed as $F_{\gamma}(\boldsymbol{p}, E, h)$, where $\boldsymbol{p}$ is a position on the Moon, $E$ is the energy of the gamma ray, and $h$ is the altitude of the SELENE spacecraft. The detection efficiency is described as $\varepsilon(\boldsymbol{p}, E)$ for a gamma ray coming from $\boldsymbol{p}$. In general, FWHM of the $P(\boldsymbol{p})=\varepsilon F_{\gamma}$ is often used as a measure of the spatial resolution.

$P(\boldsymbol{p})$ was calculated using the Geant 4 code, assuming that the GRD consists of only the Ge crystal, the BGO, and the plastic scintillators; this gives a rough estimation of
$P(\boldsymbol{p})$. The contour plots of $P(\boldsymbol{p})$ for $200 \mathrm{keV}$ and $1461 \mathrm{keV}$ are shown in Fig. 13. As one side of the Ge crystal, or the radiator side, is not covered with $\mathrm{BGO}$, as seen in Fig. 2, detection efficiencies for gamma rays incident from the radiator side are high. As a result, the contours of $P(p)$ are expanded to this side. The shape of $P(\boldsymbol{p})$ for GRD is slightly anisotropic for $1 \mathrm{MeV}$, and its FWHM is about $130 \mathrm{~km}$, viewed at the $100 \mathrm{~km}$ altitude (Hasebe et al., 2007).

One of the outstanding features of the GRS is its excellent energy resolution for gamma rays due to the employment of the high-purity Ge detector. The SELENE-GRS is the very first mission-and the only one planned in near future - to survey the lunar surface using a Ge detector. The energy resolution of the GRS $(0.4 \%$ for $662 \mathrm{keV})$ is approximately 20-25 times higher than that of scintillators used in the Apollo 15 (8.6\% for $662 \mathrm{keV}$ (Metzger et al., 1973)), Apollo 16 (9\% (Metzger et al., 1973)), Lunar Prospector (10.8\% (Feldman et al., 2004)), and Chang'E-1 missions (9\% (Sun et al., 2005)). An energy resolution of a detector is one of the most important parameters in gamma-ray spectroscopy for the determination of elemental abundances with high precision because it is inevitable for spectra obtained with a crude energy resolution to give some considerable ambiguity (systematic error) for peak analysis of gamma-ray spectra. The expected response of the Ge detector employed by the SELENE-GRS for GCR-induced lunar gamma rays is shown in Fig. 14. It is based on a spectrum of gamma rays calculated to be emitted from the Apollo 16 site (Yamashita et al., 2008), as taking the energy resolution of the GRS into account. As seen in Fig. 14, it is expected that most of gamma-ray lines from major elements will be 

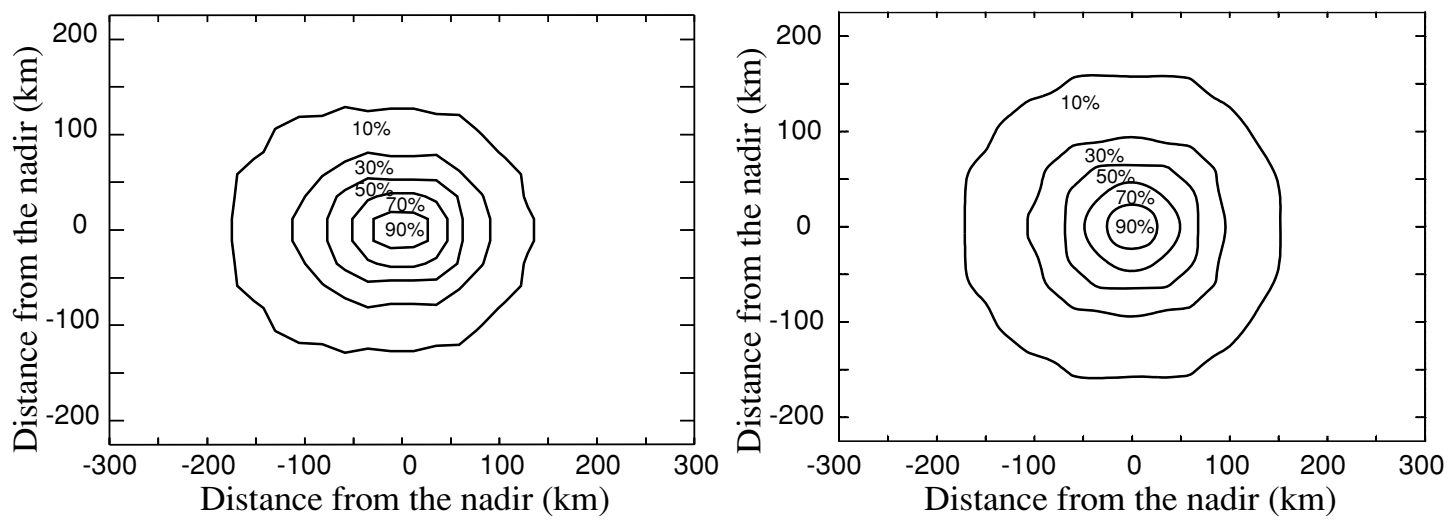

Fig. 13. Contour plots of $P=\varepsilon F_{\gamma}$ for $200 \mathrm{keV}$ (left) and $1461 \mathrm{keV}$ (right) when the GRS orbits at 100-km altitude. The contours are expanded to the left side because the Ge crystal is not shielded with BGO for this side.

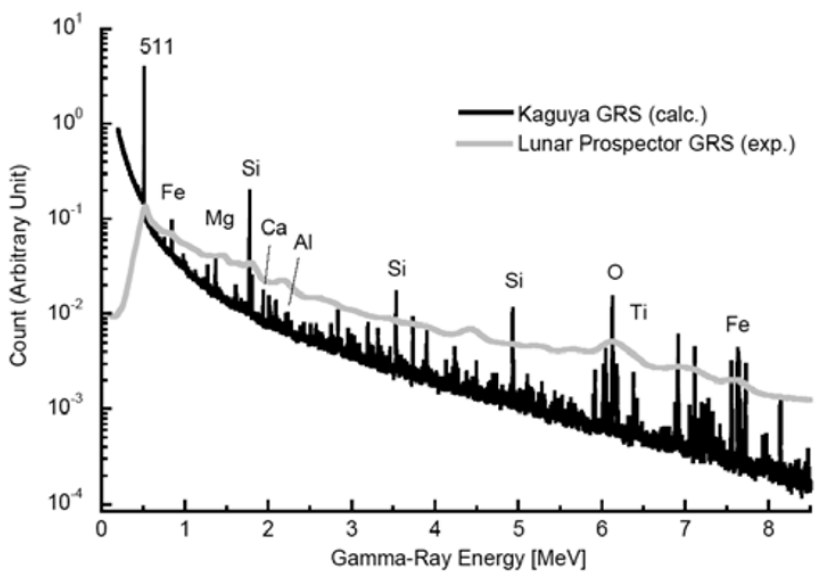

Fig. 14. Expected response of the Ge detector employed by SELENE-GRS to the GCR-induced lunar gamma rays calculated by Yamashita et al. (2008). Observational result by Lunar Prospector GRS integrated for the entire Moon by Lawrence et al. (1999) is also shown for comparison. Note that the vertical axis is in an arbitrary unit.

identified successfully. Elemental abundance maps by Lunar prospector GRS (Prettyman et al., 2006) will be globally revised by SELENE-GRS with a significantly reducing systematic errors for elemental abundances by SELENEGRS. The GRS will provide much improved global maps of $(\mathrm{H}), \mathrm{O}, \mathrm{Mg}, \mathrm{Al}, \mathrm{Si}, \mathrm{K}, \mathrm{Ca}, \mathrm{Ti}, \mathrm{Fe}, \mathrm{U}$, and Th.

Another advantage of the GRS having the excellent energy resolution is that the sensitivity to detect elements is improved under background gamma rays. Figure of merit (FOM, defined as $\left(\varepsilon_{\mathrm{det}} / \Delta E\right)^{0.5}$; Gilmore and Hemingway, 1995 ) is so used as a measure of sensitivity, where $\varepsilon_{\text {det }}$ and $\Delta E$ are a relative detection efficiency of a gamma-ray detector to a 3-inch $\mathrm{NaI}(\mathrm{Tl})$ scintillator and an energy resolution of it, respectively. The FOM for SELENE-GRS $\left(\varepsilon_{\text {det }}=0.59, \Delta E=3 \mathrm{keV}\right.$ for $\left.1333 \mathrm{keV}\right)$ is 2.9 times higher than that for Lunar Prospector GRS $\left(\varepsilon_{\mathrm{det}}=2.3\right.$, as calculated by Geant 4 using the geometry in the paper of Feldman et al. (2004; $\Delta E=103 \mathrm{keV}$ for $1333 \mathrm{keV})$. Thus, as an example, the accuracy of the abundance maps of Th in the lunar far-side where the abundance is less than 1 ppm can be greatly improved, which is a key element to understanding the early evolution of the Moon. In addition, average abundances of less-abundant elements, such as $\mathrm{Cr}$ or $\mathrm{Mn}$, on the whole of the Moon surface would be measured by SELENE-GRS.

\section{Telemetry}

The commands and telemetry of the GRS are briefly described below.

Commands: As the subsystem GPE is common to the GRS and the CPS, commands and telemetry for GRS and CPS are processed by the common CPU set. The CPU set receives commands uplinked from the ground station and sends control signals into an FPGA that executes the operation for the GRS.

The GRS has many functions controllable by commands. These include the setting of the output voltage of the HV power supply, cryocooler operation, observation mode, among others.

For the high-voltage supply units for $3.5 \mathrm{kV}$ and $1.5 \mathrm{kV}$, the output levels are controlled from $0 \mathrm{~V}$ up to the maximum voltages by 256 steps. For avoiding mis-execution of the high-voltage supply control, we adopted a "doublecommand" execution procedure. In this procedure, commands such as power-on and changing voltage level are rejected unless the enabled command for HV control is executed in advance.

In the same way, we adopted a "double-command" execution procedure for operating the cryocooler. The driving voltage to cryocooler is controlled from $0 \mathrm{~V}$ to $17 \mathrm{~V}$ by 256 steps.

For the Ge detector signal, the threshold level and the signal gain are set by commands. The threshold level is variable from $0 \mathrm{keV}$ to about $670 \mathrm{keV}$ by 256 steps. The signal gain is variable within a range of $10 \%$. For the BGO detector, two output signals from the PMTs are adjusted by individual amplifiers before summing up, of which the gains are independently controlled from 0 to $100 \%$.

For a health check of signal processing unit, test pulse input is fed into all the PAs from a pulse generator by command as the need arises. The observation mode, GEMODE, and BGO-MODE (see Section 4.2), is selected by command. The anti-coincidence processing of the Ge detector and the BGO detector is enabled or disabled by command. 
Table 1. Specifications of the gamma ray spectrometer (GRS).

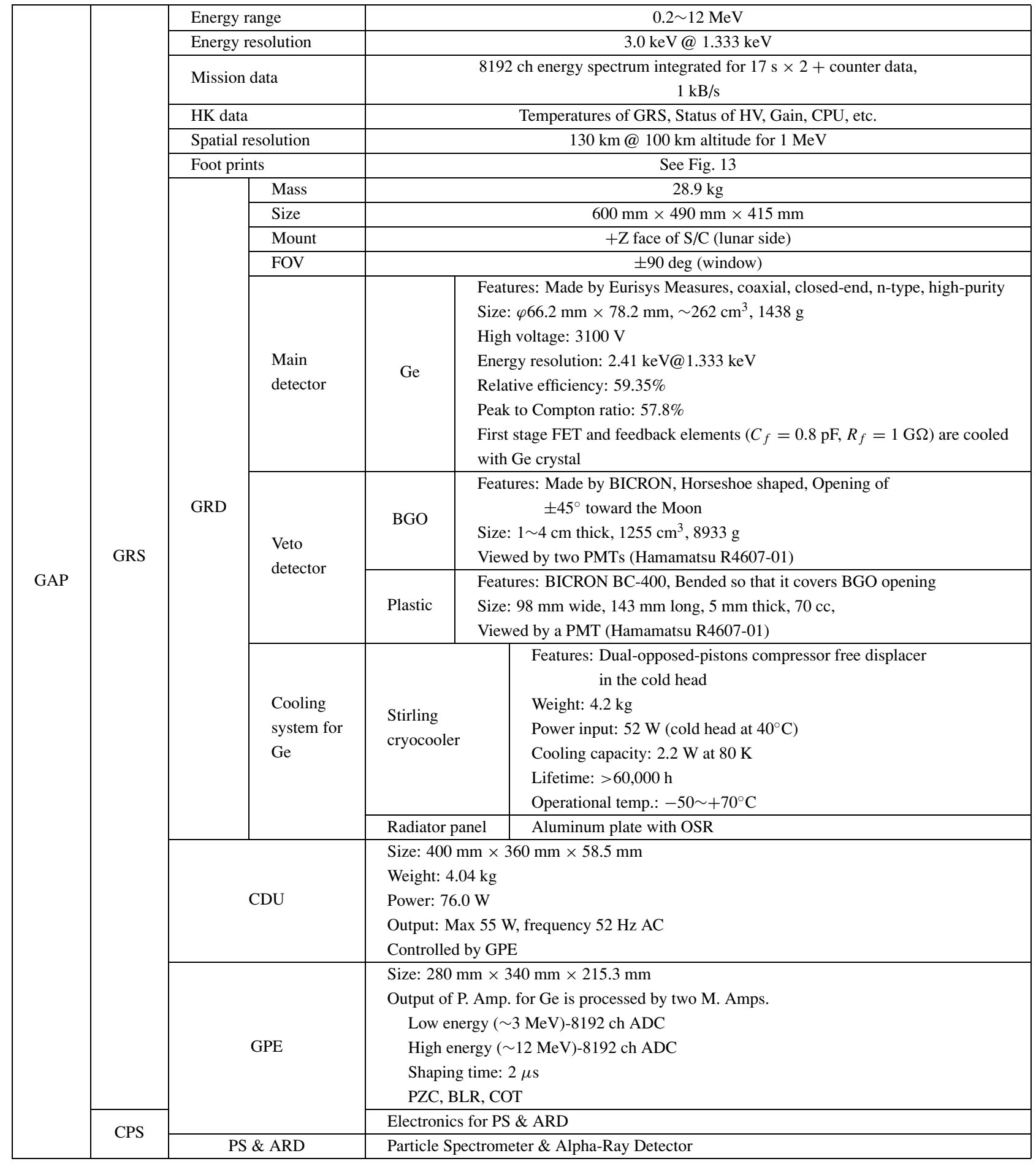

Telemetry: Telemetry data for the GRS includes scientific data and housekeeping (HK) data of the GRS. The scientific data contains dual 8192 channel pulse height analyzer (PHA) data and counter data. The PHA data exhibit two energy spectra, with energy ranges up to $3 \mathrm{MeV}$ and $12 \mathrm{MeV}$, and they are accumulated for $17 \mathrm{~s}$ and measured in time series. The PHA data contains the Ge detector data in GE-MODE (normally selected), while it contains the BGO detector data in BGO-MODE. When a gamma-ray burst event is detected (BURST-MODE), the Ge detector signals are analyzed as a PHA data for $1 \mathrm{~s}$.
The HK data collected by a FPGA on a digital circuit board show the status and the operation mode of the GRS. The HK data contain the voltage and current of the power supply, temperature, and parameters set by commands, such as HV set, anti-coincidence mode, observation mode, among others. The HK data include nine temperatures at the Ge detector, the cold tip, the cold head and the compressor of the cryocooler, the radiator, the GRD baseplate, and the electronics. These temperatures are independently measured and telemetered even though the detectors are off or the signal processing circuit boards are off. 


\section{Data Analysis and Science Product 6.1 Low level data processing}

Data received by JAXA's antenna facilities are transmitted to the SELENE Operation and Analysis Center (SOAC) at the Sagamihara Campus of ISAS/JAXA where the low level processing of SELENE data will be implemented. The data will be classified by VCID (Virtual Channel ID) into a different type of frame data, called level 0 data. The "level 0" data are classified by APID (Application Process ID) into data of different instruments, while the packets included in the data are sorted by the time index written in the header part to be the level 1 data. The real time data in "level 1" is distributed via internet, SINET (Science Information Network), and Super SINET. Scientists of the SELENE project can remotely monitor the status of SELENE in real time from outside SOAC using the Space Data Transfer Protocol (SDTP). The real time and reproduced data are archived in data storage servers at SOAC, to which the scientists can access through the network. Ancillary data of SELENE needed for scientific data analysis include information about the orbit, attitude, and ground time corresponding to the spacecraft time. All scientific data of SELENE are open only to SELENE project scientists and their co-investigators for the first year after the mission's end.

\subsection{Data analysis of SELENE-GRS}

Data analysis: The SELENE-GRS team will retrieve Level 1 data from SOAC to Waseda University and other institutes through SINET or the internet. After filtering failure packets (e.g., irregular sequence number), good-packet data are depacketized to time series data of 17 secondaccumulated 8192-channel pulse height analyzer (PHA) data and counter-data.

The observation data to be selected are based on the instrumental status of the GRS (including HV value, Ge detector temperature, and observation mode), space environment (occurrence of solar events or other particle, and gamma-ray events), and the maneuver-related events of the spacecraft (attitude, direction, orbit, and the unloading duration). The retrieved PHA data contain a time stamp from which the location of the measurement can be derived.

The PHA data are corrected for the following factors:

- Accumulation time (dead time due to onboard data processing, guard pulse, etc.);

- Solid angle subtended by the lunar surface (caused by the altitude variation from 70 to $130 \mathrm{~km}$ at the nominal altitude of $100 \mathrm{~km}$ );

- Peak drift in energy spectra due to temperature variation or other cause;

- Cosmic-ray intensity variation (using the intensity of 6.13-MeV gamma-ray line, etc.).

Since gamma rays from the lunar surface are mostly produced by galactic cosmic rays, their intensities are strongly affected by that of cosmic rays, except for gamma rays originating from natural radioactivities. The intensity of galactic cosmic rays varies as dependent the solar activity. We need correct the gamma-ray intensities (normalized to that at a certain time) using count rates of gamma rays higher than $8 \mathrm{MeV}$, the $6.13-\mathrm{MeV}$ gamma-ray line from inelastic scattering of neutrons with oxygen, and count rates of charge particles from the CPS observation.

Firstly, a time stamp enclosed in each PHA data defines its measurement location from spacecraft ancillary data. The derived location is subsequently projected to a spherical surface, and multiple PHA data in the same grid area are grouped and summed up. Each energy spectrum assigned to the same measurement location is integrated as much as possible in order to lower the minimum detection limit of the gamma-ray peaks. Then, for each spatially gridded energy spectrum, the peak fitting of gamma-ray lines will be performed to identify source nuclides and the quantitative evaluation of fluxes of incoming gamma rays.

Net counts of incoming line gamma rays will eventually be deconvoluted to determine the chemical abundance of the lunar surface materials from which they originate. The abundance of radioactive elements (K, $\mathrm{U}$, and $\mathrm{Th}$ ) will be obtained simply from reverse calculation with detection efficiency, spatial response function, absorption coefficient in lunar soil, life time, and others.

Background gamma rays: To determine the composition of the lunar surface material, we have to precisely estimate the background gamma rays originating from spacecraft because the background gamma rays can significantly interfere with those from the lunar surface. There are many processes producing background gamma rays. Gamma rays are emitted from the detector, materials surrounding the detector, and the spacecraft due to nuclear interactions similar to those that occur on the lunar surface. Thus, an understanding of sources and intensities of background gamma rays is needed in order to correctly determine the surface composition of the Moon. Seven of major background components in energy spectra to be measured in the lunar orbit are summarized as follows:

(1) Compton continuum caused by gamma rays scattered within the lunar subsurface;

(2) Compton continuum caused by a partial energy deposition of line gamma rays from the lunar surface in the detector;

(3) Lines and continuum by gamma rays promptly emitted from the spacecraft as a result of nuclear interactions;

(4) Activation of the Ge and BGO detectors and surrounding materials by cosmic rays;

(5) Natural radioactivity in the materials of GRS system, its surroundings, and the spacecraft;

(6) Continuum produced by energy deposition of charged particles in the detector;

(7) Gamma rays from astrophysical sources and other interactions of high energy particles.

Background component (1) is so inevitable that we can not essentially reduce it. The anticoincidence system of the BGO scintillator with the Ge detector can considerably decrease the background gamma rays from component (2). Background component (3) is greatly reduced by means of the BGO passive and active shield. However, a fraction of gamma rays from the spacecraft and GRS system penetrates the shield and produces the backgrounds, though the intensity depends on the energies of gamma rays. Gamma rays from aluminum and hydrogen are a particular issue of discussion. Gamma rays can also be produced by nonelastic 
Table 2. Scientific products of SELENE-GRS to L2DB.

\begin{tabular}{|l|l|l|l|}
\hline & Status & Resolution & Remarks \\
\hline $\begin{array}{l}\text { Gamma-ray } \\
\text { spectrum 1 }\end{array}$ & Energy spectrum & $\begin{array}{l}30 \times 30 \mathrm{deg} . \\
(900 \times 900 \mathrm{~km})\end{array}$ & $\begin{array}{l}\text { Uncalibrated on gain and observation } \\
\text { time. Released every 1 months }\end{array}$ \\
\hline $\begin{array}{l}\text { Gamma-ray } \\
\text { spectrum 2 }\end{array}$ & Energy spectrum & $\begin{array}{l}30 \times 30 \mathrm{deg} . \\
(900 \times 900 \mathrm{~km})\end{array}$ & $\begin{array}{l}\text { Calibrated on gain and observation time. } \\
\text { Released every 2 months }\end{array}$ \\
\hline $\begin{array}{l}\text { Gamma-ray } \\
\text { intensity map }\end{array}$ & $\begin{array}{l}\text { Intensity of line } \\
\text { gamma rays }\end{array}$ & $\begin{array}{l}2 \times 2 \mathrm{deg} . \\
(60 \times 60 \mathrm{~km})\end{array}$ & $\begin{array}{l}\text { Background subtraction } \\
(\mathrm{H}), \mathrm{O}, \mathrm{Mg}, \mathrm{Al}, \mathrm{Si}, \mathrm{K}, \mathrm{Ca}, \mathrm{Ti}, \mathrm{Fe}, \mathrm{U}, \mathrm{Th} \\
\text { Released every 3 months }\end{array}$ \\
\hline $\begin{array}{l}\text { Chemical } \\
\text { composition } \\
\text { map }\end{array}$ & Chemical composition & $\begin{array}{l}2 \times 2 \mathrm{deg} . \\
(60 \times 60 \mathrm{~km})\end{array}$ & $\begin{array}{l}(\mathrm{H}), \mathrm{O}, \mathrm{Mg}, \mathrm{Al}, \mathrm{Si}, \mathrm{K}, \mathrm{Ca}, \mathrm{Ti}, \mathrm{Fe}, \mathrm{U}, \mathrm{Th} \\
\text { Released every 6-8 months (not yet } \\
\text { determined })\end{array}$ \\
\hline
\end{tabular}

or radiative capture in the Ge detector itself, since the flux of neutrons in the SELENE orbit is high enough for such reactions within the Ge crystal. Component (5) is from the decay chains of the natural long-lived radionuclides, ${ }^{40} \mathrm{~K}$, ${ }^{232} \mathrm{Th}$, and ${ }^{238} \mathrm{U}$. It includes the daughter isotopes of ${ }^{228} \mathrm{Ac}$, ${ }^{214} \mathrm{~Pb},{ }^{214} \mathrm{Bi},{ }^{212} \mathrm{Bi},{ }^{212} \mathrm{~Pb}$, and ${ }^{208} \mathrm{Tl}$. These backgrounds were experimentally measured at the facility of spacecraft integration/testing. Component (6) is one of the largest contributions to the gamma-ray background in space, especially at higher energies. In the SELENE-GRS where BGO and plastic scintillators are used as an anti-coincidence counter, this background component is mostly rejected by the electronic veto. Component (7) is very low in intensity and further decreased by massive shields with the spacecraft body and the BGO scintillator.

\subsection{Data product and scientific cross reference}

From the viewpoint of integrated science of the Moon, it is important to confer other instrumental results for cross reference even if the results are not sufficiently calibrated. For such cross reference among instruments, SOAC has a "level 2" database (L2DB) for SELENE project scientists and their co-investigators. The "level 2" data are in global mapping form. The scientists can register their preliminary results to L2DB and they also can refer other instrumental results for their mutual reference. As shown in Table 2, the GRS team will provide L2DB with global mapping data of gamma-ray energy spectra, gamma-ray counts, and elemental concentrations of the lunar surface materials. The results registered in L2DB will be limited to SELENE project scientists and their co-investigators for the first year after the end of the nominal mission. After that, the data will be open to the public as SELENE scientific products.

The GRS, X-ray spectrometer (XRS), and Lunar Imager/SpectroMeter (LISM) in the SELENE mission will observe the elemental and mineralogical composition of the Moon. This makes it possible for the scientists to perform cross-calibration and reference. Cross-calibrations among three instruments improve the reliability of their results.

\section{Concluding Remarks and Perspectives}

An extensive review has been given in this paper on the current status of the program on the lunar gamma-ray observations, which are to be conducted by means of the GRS onboard the Japanese lunar polar orbiter SELENE launched on September 14, 2007. As have been considered earlier, various data on the gamma-ray observations to be performed in orbit are necessarily sent to us to obtain global maps of elemental composition and to access the large-scaled and local heterogeneity of the Moon. Together with the cooperative programs of the GRS observations with several other scientific instruments onboard SELENE, a lunar chemistry and geochemical terrain maps as related to the detailed horizontal and vertical structure of the Moon should give insight into the nature of the formation and evolution of the Moon. Moreover, the global maps of chemical abundance on the lunar surface to be obtained by the GRS will surely contribute to the future utilization of lunar resources in order to facilitate the progress of human activities in space.

Acknowledgments. We would like to thank the SELENE project staff at JAXA and NEC-Toshiba-Space System Ltd. for their most important supports and encouragement during the development, integration and testing of the SELENE-GRS. We also thank the staff of Ohyo Koken Kogyo Co., Ltd. for their assistance in building a part of GRS.

\section{References}

Arnold, J. R., A. E. Metzger, E. C. Anderson, and M. A. Van Dilla, Gamma rays in space, Ranger 3, J. Geophys. Res., 67, 4878-4880, 1962.

Berezhnoy, A. A., N. Hasebe, T. Hiramoto, T. Miyachi, and N. Yamashita, "Possibility of $\mathrm{H}$ and $\mathrm{S}$ detection on the Moon by SELENE mission", LPSC26th, 1210, 2003a.

Berezhnoy, A. A., N. Hasebe, B. A. Klumov, and T. Hiramoto, Possibility of the presence of $\mathrm{S}, \mathrm{SO}_{2}$ and $\mathrm{CO}_{2}$ at the poles of the Moon, Publ. Astron. Soc. Jpn., 55, 859-870, 2003b.

Bielefeld, M. J., R. C. Reedy, A. E. Metzger, J. I. Trombka, and J. R. Arnold, Surface chemistry of selected lunar regions, Proc. Lunar Sci. Conf., $7^{\text {th }}, 2661-2676,1976$.

Binder, A., Lunar Prospector: Overview, Science, 281, 1475, 1998.

Boynton, W. V., W. C. Feldman, I. G. Mitrofanov, L. G. Evans, R. C. Reedy, S. W. Squyres, R. Starr, J. I. Trombka, C. d'Uston, J. R. Arnold, P. A. J. Englert, A. E. Metzger, H. Wänke, J. Brückner, D. M. Drake, C. Shinohara, C. Fellows, D. K. Hamara, K. Harshman, K. Kerry, C. Turner, M. Ward, H. Barthe, K. R. Fuller, S. A. Storms, G. W. Thornton, J. L. Longmire, M. L. Litvak, and A. K. Ton'chev, The Mars Odyssey gamma-ray spectrometer instrument suite, Space Sci. Rev., 110, 37-83, 2004.

Campbell, D. B., B. A. Campbell, L. M. Carter, J.-L. Margot, and N. J. S. Stacy, No evidence for thick deposits of ice at the lunar south pole, Nature, 443, 835-837, 2006.

Evans, L. G., R. C. Reedy, and J. I. Trombka, Introduction to planetary remote sensing gamma ray spectroscopy, in remote geochemical analyses: Elemental and mineralogical composition, edited by C. M. Pieters and P. A. J. Englert, 167-198, Cambridge Univ. Press, New York, 1993.

Evans, L. G., R. C. Reedy, R. D. Starr, K. E. Kerry, and W. V. Boynton, Analysis of gamma-ray spectra measured by Mars Odyssey, J. Geophys. Res., 112, E3S04, 2007.

Feldman, W. C., B. L. Barraclough, K. R. Fuller, D. L. Lawrence, S. Maurice, M. C. Miler, T. H. Prettyman, and A. B. Binder, The Lunar Prospector gamma-ray and neutron spectrometers, Nucl. Instrum and Meth., A422, 562-566, 1999.

Feldman, W. C., D. L. Lawrence, R. C. Elphic, B. L. Barraclough, S. Maurice, I. Genetay, and A. B. Binder, Polar hydrogen deposits on the Moon, J. Geophys. Res., 105(E2), 4175-4195, 2000.

Feldman, W. C., S. Maurice, D. L. Lawrence, R. C. Little, S. Lawson, O. 
Gasnault, R. C. Wiens, B. L. Barraclough, R. C. Elphic, T. H. Prettyman, J. T. Steinberg, and A. B. Binder, Evidence for water ice near poles, $J$. Geophys. Res., 106(E10), 23231-23251, 2001.

Feldman, W. C., K. Ahola, B. L. Barraclough, R. D. Belian, R. K. Black, R. C. Elphic, D. T. Everett, K. R. Fuller, J. Kroesche, D. J. Lawrence, S. L. Lawson, J. L. Longmire, S. Maurice, M. C. Miller, T. H. Prettyman, S. A. Storms, and G. W. Thornton, Gamma-ray, neutron, and alpha-particle spectrometers for the Lunar Prospector mission, J. Geophys. Res., 109, E07S06, doi:10.1029/2003JE002207, 2004

Foing, B. H., M. Grande, J. Huovelin, J.-L. Josset, H. U. Keller, A. Nathues, A. Malkki, G. Noci, B. Kellett, S. Beauvivre, P. Cerroni, P. Pinet, H. Makkinen, U. Mall, M. Almeida, D. Frew, J. Volp, M. Sarkarati, D. Heather, J. Zender, P. McMannamon, and O. Camino, SMART-1 Mission: Highlights of lunar results, Lunar Planet. Sci. Conf. XXXVIII, Abstract \#1338, 2007.

Gillis, J. J., B. L. Jolliff, and R. C. Elphic, A revised algorithm for calculating $\mathrm{TiO}_{2}$ from Clementine UVVIS data: A synthesis of rock, soil, and remotely sensed $\mathrm{TiO}_{2}$ concentrations, J. Geophys. Res., 108(E2), 3-1, 2003.

Gillis, J. J., B. L. Jolliff, and R. L. Korotev, Lunar surface geochemistry: Global concentrations of $\mathrm{Th}, \mathrm{K}$, and $\mathrm{FeO}$ as derived from Lunar Prospector and Clementine data, Geochim. Cosmochim. Acta, 68(18), 3791-3805, 2004

Gilmore, G. and J. D. Hemingway, Practical gamma-ray spectrometry, John Wiley \& Sons, Chichester, 1995.

Goldsten, J. O., E. A. Rhodes, W. V. Boynton, W. C. Feldman, D. J. Lawrence, J. I. Trombka, D. M. Smith, L. G. Evans, J. White, N. W. Madden, P. C. Berg, G. A. Murphy, R. S. Gurnee, K. Strohbehn, B. D. Williams, E. D. Schaefer, C. A. Monaco, C. P. Cork, J. D. Eckels, W. O. Miller, M. T. Burks, L. B. Hagler, S. J. DeTeresa, and M. C. Witte, The MESSENGER gamma-ray and neutron spectrometer, Space Sci. Rev., 131, 339-391, doi:10.1007/s11214-007-9262-7, 2007.

Hasebe, N., N. Yamashita, O. Okudaira, S. Kobayashi, H. Yamamoto, T. Ishizaki, K. Hirano, K. Sakurai, T. Miyachi, M. Miyajima, M. Fujii, M.-N. Kobayashi, T. Takashima, E. Shibamura, O. Gasnault, S. Maurice, C. d'Uston, R. Reedy, and M. Grande, The precision gammaray spectrometer for lunar polar orbiter SELENE, J. Adv. Space Res., doi:10.1016/j.asr.2007.05.046, 2007 (in press).

Johnson, J. R., T. D. Swindle, and P. G. Lucey, Estimated solar windimplanted helium-3 distribution on the Moon, Geophys. Res. Lett., 26(3), 385-388, 1999

Jolliff, B. L., J. J. Gillis, L. A. Haskin, R. L. Korotev, and M. A. Wieczorek, Major lunar crustal terranes: Surface expressions and crust-mantle origins, J. Geophys. Res., 105(E2), 4197-4216, 2000.

Kobayashi, M.-N., N. Hasebe, T. Miyachi, T. Doke, J. Kikuchi, H. Okada, A. Oka, O. Okudaira, H. Souri, N. Yamashita, E. Shibamura, T. Kashiwagi, T. Takashima, K. Narasaki, K. Tsurumi, K. Mori, C. d’Uston, S. Maurice, M. Grande, and R. C. Reedy, High-purity germanium gammaray spectrometer with Stirling cycle cryocooler, Adv. Space Res., 30(8), 1927-1931, 2002.

Kobayashi, M.-N., N. Hasebe, T. Hiramoto, T. Miyachi, S. Murasawa, H. Okada, O. Okudaira, N. Yamashita, A. A. Berezhnoy, E. Shibamura, T. Takashima, C. d'Uston, K. Narasaki, K. Tsurumi, H. Kaneko, M. Nakazawa, K. Mori, and M. Fujii, Germanium detector with Stirling cryocooler for lunar gamma-ray spectroscopy, Nucl. Instrum. and Meth., A548, 401-410, 2005

Lawrence, D. J., W. C. Feldman, B. L. Barraclough, A. B. Binder, R. C. Elphic, S. Maurice, and D. R. Thomsen, Global Elemental Maps of the Moon: The Lunar Prospector gamma-ray spectrometer, Science, 281, 1484-1489, 1998

Lawrence, D. J., S. Maurice, and W. C. Feldman, Software Interface Specification (SIS) for the Lunar Prospector Spectrometer Planetary Data System Files, Version V001 and V002, NASA Planetary Data System, Geosciences Node (http://pds-geosciences.wustl. edu/missions/lunarp/index.htm), Washington University, St. Louis and Los Alamos Nat. Lab. Rep. LAUR-99-2753 and LAUR-99-2754, Los Alamos, N. M., 1999.

Lawrence, D. J., W. C. Feldman, B. L. Barraclough, A. B. Binder, R. C. Elphic, S. Maurice, M. C. Miller, and T. H. Prettyman, Thorium abundances on the lunar surface, J. Geophys. Res., 105, 20307-20331, 2000.

Lawrence, D. J., W. C. Feldman, R. C. Elphic, R. C. Little, T. H. Prettyman, S. Maurice, P. G. Lucey, and A. B. Binder, Iron abundances on the lunar surface as measured by the Lunar Prospector gamma-ray and neutron spectrometers, J. Geophys. Res., 107(E12), 13, 2002.

Lawrence, D. J., W. C. Feldman, R. C. Elphic, J. J. Hagerty, S. Maurice, G. W. McKinney, and T. H. Prettyman, Improved modeling of Lunar
Prospector neutron spectrometer data: Implications for hydrogen deposits at the lunar poles, J. Geophys. Res., 111(E8), E08001, 2006.

Lucey, P. G., Mineral maps of the Moon, Geophys. Res. Lett., 31, L08701, 2004

Lucey, P. G., D. T. Blewett, and B. L. Jolliff, Lunar iron and titanium abundance algorithms based on final processing Clementine UVVIS images, J. Geophys. Res., 105, 20297-20305, 2000.

Metzger, A. E. and J. R. Arnold, Gamma ray spectroscopic measurements of Mars, Appl. Optics, 9, 1289-1303, 1970.

Metzger, A. E., J. I. Trombka, L. E. Peterson, R. C. Reedy, and J. R. Arnold, Lunar surface radioactivity: Preliminary results of the Apollo 15 and Apollo 16 gamma-ray spectrometer experiments, Science, 179 , 800-803, 1973.

Metzger, A. E., R. H. Parker, J. R. Arnold, R. C. Reedy, and J. I. Trombka, Preliminary design and performance of an advanced gamma-ray spectrometer for future orbital missions, Proc. Lunar Sci. Conf., $\mathbf{6}^{\text {th }}$, 2769-2784, 1975.

Nakamura, Y., G. V. Latham, and H. J. Dorman, Apollo Lunar seismic experiment-final summary, Proc. $13^{\text {th }}$ Lunar and Planetary Sci. Conf., J. Geophys. Res., Suppl., 87, A117-A123, 1982.

Nozette, S., P. Rustan, L. P. Pleasance, J. F. Kordas, I. T. Lewis, H. S Park, R. E. Priest, D. M. Horan, P. Regeon, C. L. Lichtenberg, E. M. Shoemaker, E. M. Eliason, A. S. McEwen, M. S. Robinson, P. D. Spudis, C. H. Acton, B. J. Buratti, T. C. Duxbury, D. N. Baker, B. M. Jakosky, J. E. Blamont, M. P. Corson, J. H. Resnick, C. J. Rollins, M. E. Davies, P. G. Lucey, E. Malaret, M. A. Massie, C. M. Pieters, R. A. Reisse, R. A. Simpson, D. E. Smith, T. C. Sorenson, R. W. Vorder Breugge, and M. T. Zuber, The Clementine mission to the Moon-Scientific overview, Science, 266, 1835-1839, 1994.

Nozette, S., P. D. Spudis, M. S. Robinson, D. B. J. Bussey, C. Lichtenberg, and R. Bonner, Integration of lunar polar remote-sensing data sets: Evidence for ice at the lunar south pole, J. Geophys. Res., 106, 23253 23266, 2001

Prettyman, T. H., J. J. Hagerty, R. C. Elphic, W. C. Feldman, D. J. Lawrence, G. W. McKinney, and D. T. Vaniman, Elemental composition of the lunar surface: Analysis of gamma ray spectroscopy data from Lunar Prospector, J. Geophys. Res., 111, E12007, 2006.

Reedy, R. C., Planetary gamma-ray spectroscopy, Proc. Lunar Sci. Conf., 9th, 2961-2984, 1978.

Reedy, R. C., J. R. Arnold, and J. I. Trombka, Expected gamma ray emission spectra from the lunar surface as a function of chemical composition, J. Geophys. Res., 78, 5347-5866, 1973.

Sasaki, S., Y. Iijima, K. Tanaka, M. Kato, M. Hashimoto, H. Mizutani, and Y. Takizawa, The SELENE mission: Goals and status, Adv. Space Res., 31, 2335-2340, 2003.

Shearer, C. K. and J. J. Papike, Magmatic evolution of the Moon, Am. Mineral., 84, 1469-1497, 1999.

Solomon, S. C., R. L. McNutt, R. E. Gold, M. H. Acuña, D. N. Baker, W. V. Boynton, C. R. Chapman, A. F. Cheng, G. Gloeckler, J. W. Head III, S. M. Krimigis, W. E. McClintock, S. L. Murchie, S. J. Peale, R. J. Phillips, M. S. Robinson, J. A. Slavin, D. E. Smith, R. G. Strom, J. I. Trombka, and M. T. Zuber, The MESSENGER mission to Mercury: Scientific objectives and implementation, Planet. Space Sci., 49, 14451465,2001

Sun, H. X. and S. W. Dai, Mission objectives and payloads for the first lunar exploration of China, Acta Astronautica, 57, 561-565, 2005.

Surkov, Yu. A., Nuclear physical methods of analysis in lunar and planetary investigations, Isotopenpraxis, 20, 321-329, 1984.

Takeda, H., A. Yamaguchi, D. D. Bogard, Y. Karouji, M. Ebihara, M. Ohtake, K. Saiki, and T. Arai, Magnesian anorthosites and a deep crustal rock from the farside crust of the Moon, Earth Planet. Sci. Lett., 247, 171-184, 2006

Warren, P. H., "New" lunar meteorites: Imprecations for composition of the global lunar surface, lunar crust, and the bulk Moon, Meteor. Planet. Sci., 40, 477-506, 2005.

Yamashita, N., N. Hasebe, T. Miyachi, M. Kobayashi, O. Okudaira, S. Kobayashi, T. Ishizaki, K. Sakurai, M. Miyajima, R. C. Reedy, C. d'Uston, S. Maurice, and O. Gasnault, Complexities of gamma-ray line intensities from the lunar surface, Earth Planets Space, 60, this issue, 313-319, 2008

N. Hasebe, E. Shibamura, T. Miyachi, T. Takashima, M. Kobayashi, O Okudaira, N. Yamashita, S. Kobayashi (e-mail: shingo@ruri.waseda.jp), T. Ishizaki, K. Sakurai, M. Miyajima, M. Fujii, K. Narasaki, S. Takai, K. Tsurumi, H. Kaneko, M. Nakazawa, K. Mori, O. Gasnault, S. Maurice, C. d'Uston, R. C. Reedy, and M. Grande 